\title{
Performance of Equal Power Allocation in Multiuser OFDM-Based Cognitive Radio Systems
}

\author{
Yonghong Zhang and Cyril Leung \\ Department of Electrical and Computer Engineering, The University of British Columbia, Vancouver, BC, Canada V6T $1 Z 4$
}

Correspondence should be addressed to Cyril Leung, cleung@ece.ubc.ca

Received 22 July 2008; Accepted 15 September 2008

Recommended by Adam Panagos

The performance difference between a simple equal subchannel power allocation and the optimal water-filling subchannel power allocation schemes is studied for a multiuser OFDM-based cognitive radio (CR) system. It is shown that this difference depends on the average subchannel gain variations among the CR users as well as the activity levels of the primary users.

Copyright (C) 2008 Y. Zhang and C. Leung. This is an open access article distributed under the Creative Commons Attribution License, which permits unrestricted use, distribution, and reproduction in any medium, provided the original work is properly cited.

\section{Introduction}

Cognitive radio (CR) [1-3] is a concept which can potentially alleviate the pending spectrum shortage crisis. As discussed in [2], orthogonal frequency division multiplexing (OFDM) is an attractive modulation candidate for CR systems. It is well known that the optimal solution to the problem of determining the capacity of a set of $M$ parallel additive white Gaussian noise (AWGN) subchannels, each of which may have a different noise power level, subject to a total input signal power constraint, has a nice water-filling interpretation [4]. We will refer to this as optimal water-filling (OWF). In OWF, the signal powers allocated to different subchannels are in general different and no power is allocated to "silent" subchannels on which the noise power exceeds a certain threshold (water level). The scheme in which the total available signal power is shared equally among all (silent and nonsilent) subchannels is referred to as plain equal power allocation (PEPA).

Simulation results in [5] indicate that the difference between OWF and PEPA is quite small in a multiple user system with Rayleigh fading when each subchannel is assigned to the user with the best channel quality for that subchannel. Analytical results in [6] show that the performance difference between OWF and PEPA decreases with the number of users and average signal-to-noise ratio (SNR). In this paper, we study the performance difference between PEPA and OWF in a multiuser OFDM-based CR system. It is found that PEPA performs almost as well as OWF when there is little variation in CR user (CRU) average subchannel gains or PU activity level is high.

\section{System Model}

We consider a CR system with a total bandwidth of $W \mathrm{~Hz}$ and $L$ PUs; PU $l, l=1,2, \ldots, L$ has a bandwidth allocation of $W_{l} \mathrm{~Hz}$. Frequency bands carrying PU signals are referred to as active; nonactive bands are also termed spectrum holes. In order to reduce the mutual interference between secondary CRUs and PUs to acceptable levels, some subchannels adjacent to active PU bands are not used by the CRUs.

We are interested in downlink transmissions from one CR base station (CRBS) to $K$ CRUs. It is assumed that the CRBS and the CRUs are able to accurately locate the spectrum holes. The system bandwidth of $W \mathrm{~Hz}$ can accommodate $M$ OFDM subbands (or subchannels), each with noise power $\sigma_{0}^{2}$. Interference among the subchannels is assumed to be negligible.

The system is time-slotted with a slot duration equal to an OFDM symbol duration $\left(T_{s}\right)$. The subchannels are modelled in discrete time, with the gain for subchannel $m$ and time slot $t$ from the CRBS to CRU $k$ denoted by $\sqrt{g_{k, m}^{t}}$. For simplicity, it is assumed that for any given value of $k,\left\{g_{k, m}, m=1,2, \ldots, M\right\}$ are identically distributed random variables (RVs) with a common probability function 
(pdf) and cumulated distribution function (cdf) denoted as $f_{G_{k}}\left(g_{k}\right)$ and $F_{G_{k}}\left(g_{k}\right)$, respectively.

At each time slot $t$, each subchannel within $\mathcal{M}_{t}$, the set of available subchannels at time slot $t$, can be used by the CRBS to transmit to at most one CRU. We use $f_{G}(g)$ and $F_{G}(g)$ to denote the pdf and the cdf of the selected CRUs, respectively. The number $\left(r_{m}^{t}\right)$ of bits per OFDM symbol which can be supported by subchannel $m$ in time slot $t$ is given by [7]

$$
r_{m}^{t}=\log _{2}\left(1+\frac{g_{m}^{t} s_{m}^{t}}{\Gamma \sigma_{0}^{2}}\right)
$$

where $g_{m}^{t}$ is the subchannel gain of the selected CRU, $s_{m}^{t}$ is the power allocated to subchannel $m$ at time slot $t$, and $\Gamma$ is an SNR gap parameter which indicates how far the system is operating from capacity. The available power constraint implies that

$$
\sum_{m \in \mathcal{M}_{t}} s_{m}^{t} \leq S \quad \forall t
$$

where $S$ is the total power per time slot.

The availability of a PU band is modelled by a two-state Markov chain. During a time slot $t$, a PU band can be in one of two modes: active or inactive [8]. A PU band can change mode once every $T_{\text {state }}$ slots. At a transition time, the probability of a PU band changing from active to inactive mode is $1-p_{a}$, and the probability of changing from inactive to active mode is $1-p_{n}$. The number $\left(l_{\mathrm{CR}, t}\right)$ of available PU bands at time slots $\{t, t=1,2, \ldots\}$ then forms a Markov chain, with a transition probability matrix $\mathbf{Q}=\left\{q_{i j}\right\}, i, j=$ $0,1,2, \ldots, L$, where state $i$ corresponds to the event that the number of available PU bands is equal to $i$ and the probability $\left(q_{i j}\right)$ of moving from state $i$ to state $j$ is given by

$$
q_{i j}=\sum_{n=0}^{L}\left(\begin{array}{l}
i \\
n
\end{array}\right)\left(1-p_{n}\right)^{n} p_{n}^{i-n}\left(\begin{array}{c}
L-i \\
n-i+j
\end{array}\right)\left(1-p_{a}\right)^{n-i+j} p_{a}^{L-j-n}
$$

The steady-state probability column vector $\Pi=$ $\left(\pi_{0}, \pi_{1}, \ldots, \pi_{L}\right)^{T}$ is given by [9]

$$
\Pi=\mathbf{U}^{-1} \mathbf{V},
$$

where

$$
\mathbf{U}=\left[\begin{array}{ccccc}
1, & 1, & 1, & \cdots & 1 \\
0, & 1-q_{00}+q_{10}, & 1-q_{00}+q_{20}, & \cdots & 1-q_{00}+q_{L 0} \\
0, & q_{01}+1-q_{11}, & q_{01}-q_{21}, & \cdots & q_{01}-q_{L 1} \\
\cdots & \ldots & \cdots & \cdots & \cdots \\
0, & q_{0 L}-q_{1 L}, & q_{0 L}-q_{2 L}, & \cdots & q_{0 L}+1-q_{L L}
\end{array}\right],
$$

and $\mathbf{V}=\left(1,1-q_{00}, q_{01}, \ldots, q_{0 L}\right)^{T}$. If each PU band can accommodate an equal number $(M / L)$ of subchannels, the probability of having $m$ available subchannels is

$$
b_{m}= \begin{cases}\pi l, & \text { if } m=\frac{l M}{L}, l=0,1,2, \ldots, L \\ 0, & \text { otherwise }\end{cases}
$$

\section{Achievable Bit Rates for OWF and PEPA}

Although OWF provides the optimal solution for subchannel power allocation, it is more complex-to-implement than PEPA. We now derive expressions to compare the bit rates achievable by the two schemes.

\subsection{Upper Bounds on Achievable Bit Rate for OWF}

Assuming that OWF is applied to the $m_{\mathrm{CR}, t}$ subchannel gains of the selected CRUs at time slot $t$, the average bit rate (ABR) $\left(B_{\mathrm{OWF}} \triangleq \lim _{T \rightarrow \infty} B_{\mathrm{OWF}}^{(T)}\right)$ is given by [4]

$$
B_{\mathrm{OWF}}^{(T)}=\frac{1}{T_{s} T} \sum_{t=1}^{T} \sum_{m \in \mathcal{M}_{t}: \Gamma \sigma_{0}^{2} / g_{m}^{t} \leq L_{\mathrm{OWF}}^{t}} \log _{2}\left(\frac{L_{\mathrm{OWF}}^{t} g_{m}^{t}}{\Gamma \sigma_{0}^{2}}\right) .
$$

In (7), $\Gamma \sigma_{0}^{2} / g_{m}^{t}$ can be viewed as the equivalent noise power on subchannel $m$ and $L_{\mathrm{OWF}}^{t}$ is the water level at time $t$.

Let $\mathcal{T}_{i}$ be the set of time slots with $m_{\mathrm{CR}, t}=i$, and $T_{i}$ be the number of elements in set $\mathcal{T}_{i}$. Grouping the time slots with $m_{\mathrm{CR}, t}=i$, we can rewrite (7) as

$$
B_{\mathrm{OWF}}^{(T)}=\frac{1}{T_{s} T} \sum_{i=1}^{M} \sum_{t_{i} \in \mathcal{T}_{i}} \sum_{m \in \mathcal{M}_{t_{i}}: \Gamma \sigma_{0}^{2} / g_{m}^{t_{i}} \leq L_{\mathrm{OWF}, i}^{t_{i}}} \log _{2}\left(\frac{L_{\mathrm{OWF}, i}^{t_{i}} g_{m}^{t_{i}}}{\Gamma \sigma_{0}^{2}}\right),
$$

where $L_{\mathrm{OWF}, i}^{t_{i}}$ is the water level at time slot $t_{i}$.

When $m_{\mathrm{CR}, t}=i$, the ABR if OWF is applied at each time slot $t_{i}$ is smaller than that if OWF is applied in one shot to all the $i T_{i}$ subchannel gains of the selected CRUs over the $T_{i}$ time slots, that is,

$$
B_{\mathrm{OWF}}^{(T)} \leq \frac{1}{T_{s} T} \sum_{i=1}^{M} \sum_{t_{i} \in \mathcal{T}_{i}} \sum_{m \in \mathcal{M}_{t_{i}}: \Gamma \sigma_{0}^{2} / g_{m}^{t_{i}} \leq L_{\mathrm{OWF}, i}^{\left(T_{i}\right)}} \log _{2}\left(\frac{L_{\mathrm{OWF}, i}^{\left(T_{i}\right)} g_{m}^{t_{i}}}{\Gamma \sigma_{0}^{2}}\right),
$$

where $L_{\mathrm{OWF}, i}^{\left(T_{i}\right)}$ is the global water level for set $\mathcal{T}_{i}$.

Letting $T_{i}=b_{i} T$, and taking the limit as $T \rightarrow \infty$, we have

$$
B_{\mathrm{OWF}} \leq \frac{1}{T_{s}} \sum_{i=1}^{M} i b_{i} \int_{\Gamma \sigma_{0}^{2} / L_{\mathrm{OWF}, i}^{(\infty)}}^{\infty} \log _{2}\left(\frac{L_{\mathrm{OWF}, i}^{(\infty)}}{\Gamma \sigma_{0}^{2}}\right) f_{G}(g) d g .
$$

In (9), $L_{\mathrm{OWF}, i}^{\left(T_{i}\right)}$, is lower than the level calculated by including all subchannels at time slots $t \in \mathcal{T}_{i}$ because there may exist some subchannels with above water level noises. Therefore,

$$
L_{\mathrm{OWF}, i}^{\left(T_{i}\right)} \leq \frac{\sum_{t \in \mathcal{T}_{i}} \sum_{m \in \mathcal{M}_{t}}\left(\Gamma \sigma_{0}^{2} / g_{m}^{t}\right)}{i T_{i}}+\frac{S}{i} .
$$

Taking the limit as $T_{i} \rightarrow \infty$ in (11) yields

$$
L_{\mathrm{OWF}, i}^{(\infty)} \leq E\left\{\frac{1}{G}\right\} \Gamma \sigma_{0}^{2}+\frac{S}{i} .
$$


Substituting for the two occurrences of $L_{\mathrm{OWF}, i}^{(\infty)}$ in $(10)$ by the RHS of (12), we obtain

$$
\begin{aligned}
& B_{\mathrm{OWF}} \\
& \leq \frac{1}{T_{s}} \sum_{i=1}^{M} i b_{i}\left[\left(1-F_{G}\left(\frac{1}{L_{i}}\right)\right) \log _{2}\left(L_{i}\right)+\int_{1 / L_{i}}^{\infty} \log _{2}(g) f_{G}(g) d g\right],
\end{aligned}
$$

where $L_{i}=\mathbf{L}_{i}$ and $\mathbf{L}_{i} \triangleq E\{1 / G\}+S /\left(i \Gamma \sigma_{0}^{2}\right)$.

The upper bound for $L_{\mathrm{OWF}, i}^{(\infty)}$ in (12) can be quite loose since it includes every subchannel, regardless of its equivalent noise power $\Gamma \sigma_{0}^{2} / g_{m}^{t}$. As $g_{m}^{t} \rightarrow 0, \Gamma \sigma_{0}^{2} / g_{m}^{t}$ increases without bound. Consequently, the bound in (13) is also loose. We obtain a tighter bound by turning off any subchannel $m \in$ $\mathcal{M}_{t}$ for which $\Gamma \sigma_{0}^{2} / g_{m}^{t}$ is greater than the RHS of (12), that is, we consider only the subchannels for which $g_{m}^{t}>1 / \mathbf{L}_{i}$ in calculating the water levels. The resulting water level is still higher than $L_{\mathrm{OWF}, i}^{(\infty)}$ so that

$$
L_{\mathrm{OWF}, i}^{(\infty)} \leq \frac{\Gamma \sigma_{0}^{2} \int_{1 / \mathbf{L}_{i}}^{\infty}(1 / g) f_{G}(g) d g+S / i}{1-F_{G}\left(1 / \mathbf{L}_{i}\right)} .
$$

Substituting $L_{\mathrm{OWF}, i}^{(\infty)}$ in (10) by the RHS of (14), we obtain a tighter bound, namely, (13) with

$$
L_{i}=\frac{\int_{1 / \mathbf{L}_{i}}^{\infty}(1 / g) f_{G}(g) d g+S /\left(i \Gamma \sigma_{0}^{2}\right)}{1-F_{G}\left(1 / \mathbf{L}_{i}\right)} .
$$

\subsection{Achievable Bit Rate for PEPA}

The ABR for PEPA is $B_{\mathrm{PEPA}} \triangleq \lim _{T \rightarrow \infty} B_{\mathrm{PEPA}}^{(T)}$, where

$$
B_{\mathrm{PEPA}}^{(T)}=\frac{1}{T_{s} T} \sum_{t=1}^{T} \sum_{m \in \mathcal{M}_{t}} \log _{2}\left(1+\frac{S g_{m}^{t}}{m_{\mathrm{CR}, t} \Gamma \sigma_{0}^{2}}\right) .
$$

Grouping the time slots with $m_{\mathrm{CR}, t}=i$, we have

$$
B_{\mathrm{PEPA}}^{(T)}=\frac{1}{T_{s} T} \sum_{i=1}^{M} \sum_{t \in \mathcal{T}_{i}} \sum_{m \in \mathcal{M}_{t}} \log _{2}\left(1+\frac{S g_{m}^{t}}{i \Gamma \sigma_{0}^{2}}\right) .
$$

Letting $T_{i} \rightarrow \infty$, we obtain

$$
B_{\mathrm{PEPA}}=\frac{1}{T_{s}} \sum_{i=1}^{M} i b_{i} E\left\{\log _{2}\left(1+\frac{S}{i \Gamma \sigma_{0}^{2}} G\right)\right\} .
$$

\section{Rayleigh Fading Channel}

In Section 3, we studied the ABR for OWF and PEPA for arbitrary pdf's and cdf's of the subchannel gains of the selected CRUs. In this section, we obtain the pdf and cdf of the subchannel gains of the selected CRUs for two different subchannel allocation strategies. The subchannel gains of the CRUs are assumed to be Rayleigh-distributed, that is, the power gains are exponentially distributed.

\subsection{Opportunistic Subchannel Assignment}

Suppose that at each time $t$, each of the $M$ subchannels is assigned to the CRU with the highest gain for that subchannel. If the average subchannel power gains for all CRUs are equal, the pdf of the power gain for the CRU assigned to any subchannel is readily obtained using a standard result in order statistics [10], that is,

$$
f_{G}(g)=K\left[1-e^{-g / E\{G\}}\right]^{K-1} \frac{e^{-g / E\{G\}}}{E\{G\}}
$$

with corresponding cdf

$$
F_{G}(g)=\left[1-e^{-g / E\{G\}}\right]^{K} .
$$

\subsection{A Fairer Subchannel Assignment Scheme}

If the average subchannel gains for CRUs are quite different, assigning a subchannel to the CRU with the highest gain may be too unfair to CRUs with poor average subchannel gains. A fairer scheme [11] is to select, for each subchannel, the CRU with the best channel gain relative to its own mean gain, that is,

$$
k^{*}(t)=\underset{k}{\operatorname{argmax}} \frac{g_{k, m}(t)}{E\left\{G_{k}\right\}} .
$$

The distribution of a CRU's subchannel gain relative to its own mean is exponential with a mean of 1 . Thus, the probability of selecting CRU $i$ is $1 / K$, that is, $P\left(k^{*}=i\right)=$ $1 / K, i=1,2, \ldots, K$. The cdf of the power gain of the selected CRU for a subchannel is

$$
\begin{aligned}
F_{G}(g) & =P(G \leq g) \\
& =\sum_{i=1}^{K} P\left(G \leq g \mid k^{*}=i\right) P\left(k^{*}=i\right) \\
& =\frac{1}{K} \sum_{i=1}^{K} \prod_{j=1}^{K} P\left(G_{j} \leq \frac{g E\left\{G_{j}\right\}}{E\left\{G_{i}\right\}}\right) \\
& =\frac{1}{K} \sum_{i=1}^{K}\left(1-e^{-g / E\left\{G_{i}\right\}}\right)^{K} .
\end{aligned}
$$

The corresponding pdf is

$$
f_{G}(g)=\sum_{i=1}^{K}\left(1-e^{-g / E\left\{G_{i}\right\}}\right)(K-1) \frac{e^{-g / E\left\{G_{i}\right\}}}{E\left\{G_{i}\right\}} .
$$

\section{Numerical Results}

To compare the ABR for OWF and PEPA in a multiuser OFDM-based CR system, the expressions in (7), (18), and (13) with $L_{i}$ equal to the RHS of (15) are evaluated. The two subchannel allocation strategies in Sections 4.1 and 4.2, hereafter referred to as Case A and Case B, respectively, are considered. In Case A, the average subchannel power gain for each CRU is chosen as $2 \times 10^{-13}$. In Case $B$, we increase the 


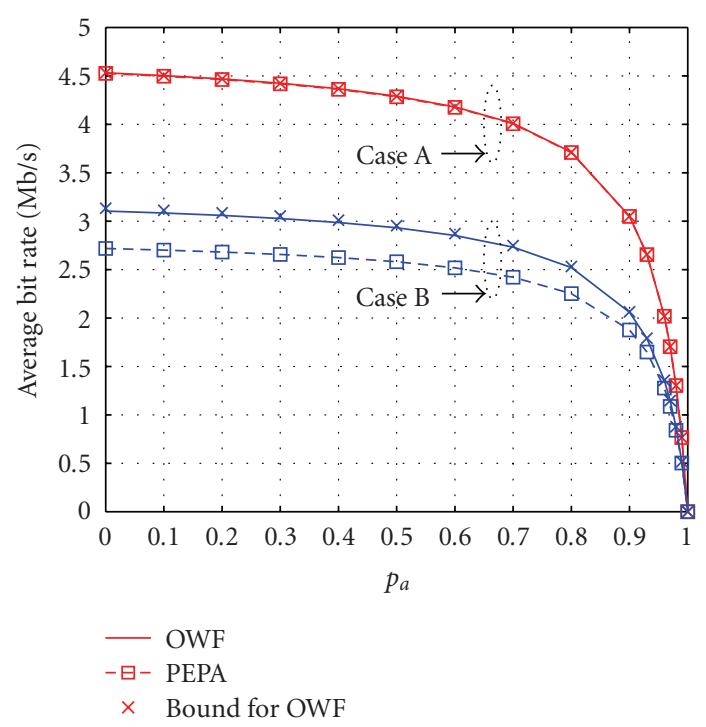

FIgURE 1: ABR as a function of $p_{a}$ for OWF and PEPA with $K=6$.

number of CRUs by six at a time. The average subchannel power gains of the six CRUs are chosen as follows: one with value $10^{-12}$, two with value $10^{-13}$ and three with value $10^{-14}$. The resulting overall average subchannel gain for the six CRUs is $2 \times 10^{-13}$. In our calculations, we also use the following parameter values: $\Gamma=1, \sigma_{0}^{2}=10^{-16}, S=0.1 \mathrm{~W}$, $W=2 \mathrm{MHz}, W_{l}=250 \mathrm{kHz}, l \in\{1,2, \ldots, L\}, L=8, M=64$, $p_{n}=0.9$, and $T_{s}=40 \mu \mathrm{s}$.

Figure 1 shows the ABR for OWF and PEPA as a function of $p_{a}$ for $K=6$ CRUs. For both cases, the ABR for OWF and PEPA decreases with $p_{a}$ due to the reduced number of available subchannels. In Case $\mathrm{A}$, the improvement of OWF over PEPA is $0.1 \%$ at $p_{a}=0.1$ and $0.05 \%$ at $p_{a}=0.9$. In Case B, the improvement of OWF over PEPA is $14 \%$ at $p_{a}=0.1$ and $2 \%$ at $p_{a}=0.99$. The difference between OWF and PEPA decreases with $p_{a}$ because with a fixed total power, the average SNR for the available subchannels increases. The difference between OWF and PEPA is known to decrease with average SNR [6]. For both cases, the proposed upper bound for OWF is very close to the actual OWF curve and the difference decreases with $p_{a}$. The results show that the relative performance of PEPA depends on the activity level of the PUs and the variations in average subchannel gains among the CRUs.

The ABRs for OWF and PEPA were also determined as a function of the number $(K)$ of CRUs. For both cases, the ABRs of OWF and PEPA increase with $K$ as a result of multiuser diversity. The ABR difference between OWF and PEPA in Case A is negligible; in Case B, the improvement of OWF over PEPA is $9 \%$ for $K=6$ and $2 \%$ for $K=48$.

\section{Conclusions}

The performance difference between the PEPA and OWF subcarrier power allocation schemes in a multiuser OFDMbased CR system was studied. A proposed upper bound for OWF was shown to be tight. When the PU activity is high or the CRU average gains are similar, the simpler PEPA scheme suffers little loss relative to OWF.

\section{References}

[1] J. Mitola III and G. Q. Maguire Jr., "Cognitive radio: making software radios more personal," IEEE Personal Communications, vol. 6, no. 4, pp. 13-18, 1999.

[2] T. A. Weiss and F. K. Jondral, "Spectrum pooling: an innovative strategy for the enhancement of spectrum efficiency," IEEE Communications Magazine, vol. 42, no. 3, pp. S8-S14, 2004.

[3] S. Haykin, "Cognitive radio: brain-empowered wireless communications," IEEE Journal on Selected Areas in Communications, vol. 23, no. 2, pp. 201-220, 2005.

[4] R. Gallager, Information Theory and Reliable Communication, John Wiley \& Sons, New York, NY, USA, 1968.

[5] J. Jang and K. B. Lee, "Transmit power adaptation for multiuser OFDM systems," IEEE Journal on Selected Areas in Communications, vol. 21, no. 2, pp. 171-178, 2003.

[6] Y. Zhang and C. Leung, "Performance of equal power subchannel loading in multiuser OFDM systems," in Proceedings of the IEEE Pacific Rim Conference on Communications, Computers and Signal Processing (PACRIM '07), pp. 526-529, Victoria, Canada, August 2007.

[7] A. J. Goldsmith and S.-G. Chua, "Variable-rate variablepower MQAM for fading channels," IEEE Transactions on Communications, vol. 45, no. 10, pp. 1218-1230, 1997.

[8] H. Su and X. Zhang, "Cross-layer based opportunistic MAC protocols for QoS provisionings over cognitive radio wireless networks," IEEE Journal on Selected Areas in Communications, vol. 26, no. 1, pp. 118-129, 2008.

[9] P. G. Hoel, S. C. Port, and C. J. Stone, Introduction to Stochastic Processes, Houghton Mifflin, Boston, Mass, USA, 1972.

[10] H. A. David and H. N. Nagaraja, Order Statistics, John Wiley \& Sons, New York, NY, USA, 3rd edition, 2003.

[11] S. Ryu, B.-H. Ryu, H. Seo, M. Shin, and S. Park, "Wireless packet scheduling algorithm for OFDMA system based on time-utility and channel state," ETRI Journal, vol. 27, no. 6, pp. 777-787, 2005. 

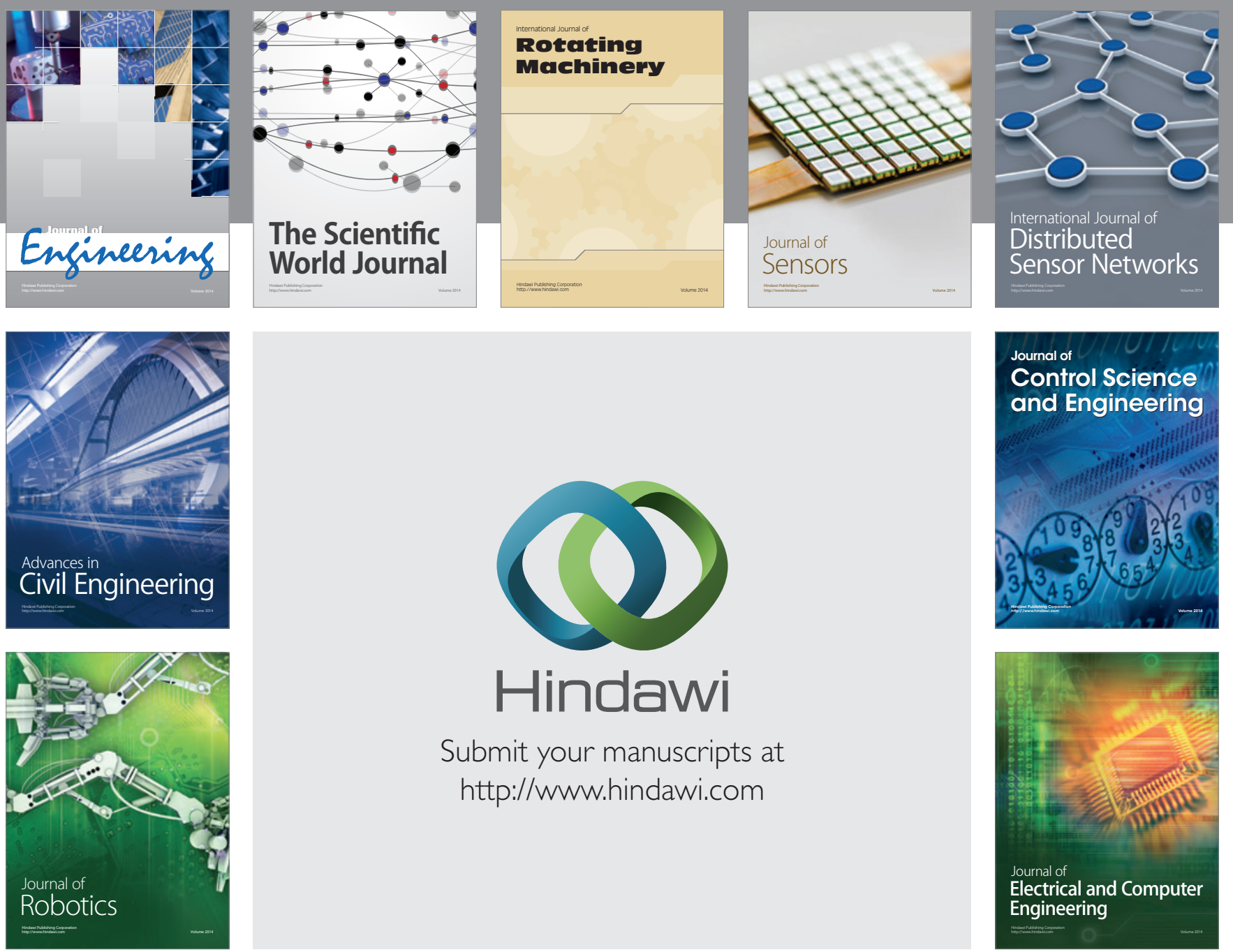

Submit your manuscripts at

http://www.hindawi.com
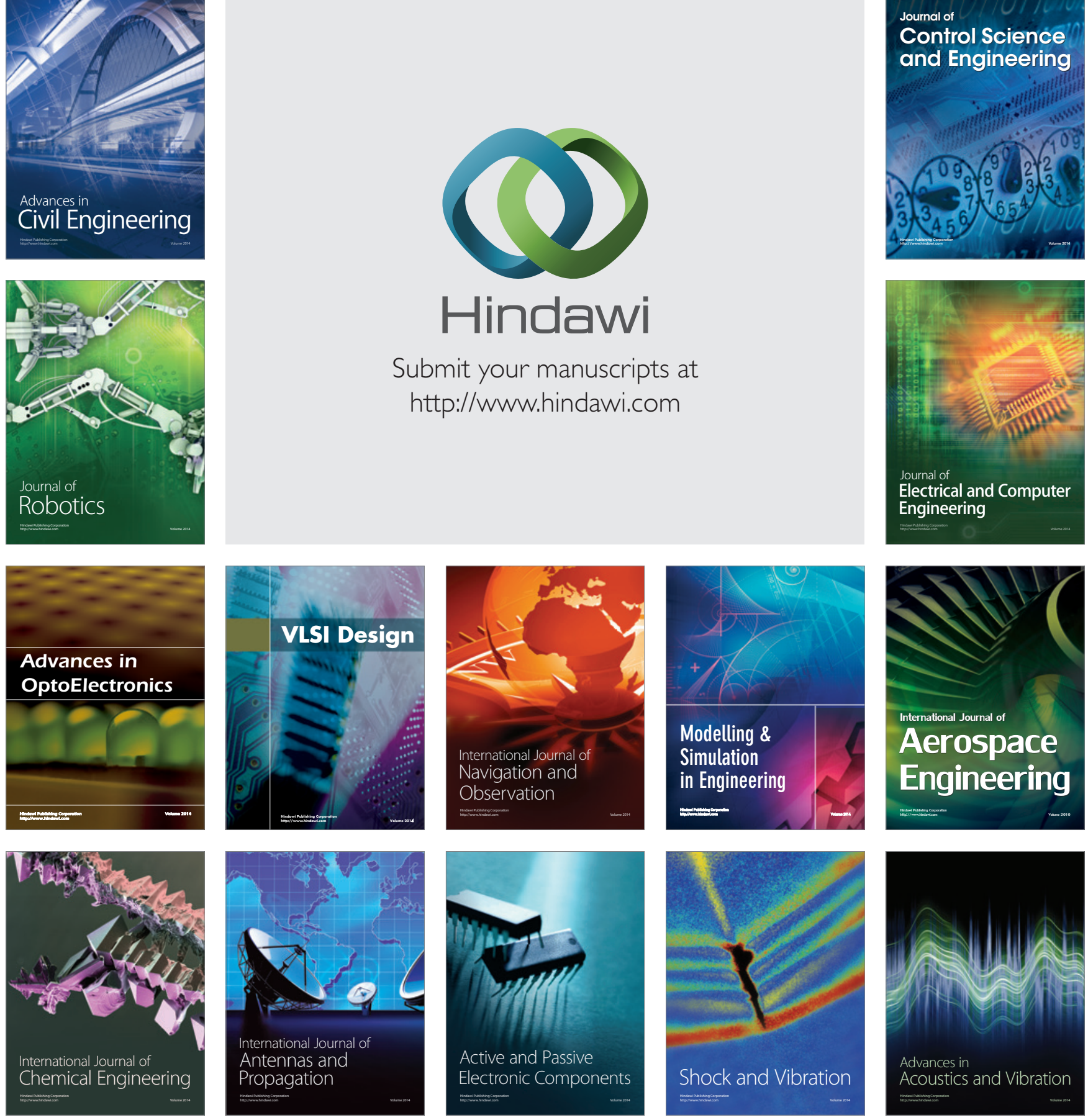